\title{
Macroscale structural changes of thylakoid architecture during high light acclimation in Chlamydomonas reinhardtii
}

\author{
Mimi Broderson ${ }^{1,2}$, Krishna K. Niyogi ${ }^{1,2,3}$, and Masakazu Iwai ${ }^{1,3 *}$ \\ ${ }^{1}$ Department of Plant and Microbial Biology, University of California, Berkeley, CA 94720, USA \\ ${ }^{2}$ Howard Hughes Medical Institute, University of California, Berkeley, CA 94720, USA \\ ${ }^{3}$ Molecular Biophysics and Integrated Bioimaging Division, Lawrence Berkeley National Laboratory, \\ Berkeley, CA 94720, USA
}

*For correspondence: miwai@lbl.gov

Keywords: Airyscan microscopy, live-cell imaging, photosynthesis, thylakoid structure, membrane remodeling, photoacclimation, Chlamydomonas reinhardtii 


\begin{abstract}
Photoprotection mechanisms are ubiquitous among photosynthetic organisms. The photoprotection capacity of the green alga Chlamydomonas reinhardtii is correlated with protein levels of stress-related light-harvesting complex (LHCSR) proteins, which are strongly induced by high light (HL). However, the dynamic response of overall thylakoid structure during acclimation to growth in HL has not been characterized. Here, we combined live-cell super-resolution microscopy and analytical membrane subfractionation to investigate macroscale structural changes of thylakoid membranes during HL acclimation in $C$. reinhardtii. Subdiffraction-resolution bioimaging revealed that overall thylakoid structures became thinned and shrunken during HL acclimation. The stromal space around the pyrenoid also became enlarged. Analytical density-dependent membrane fractionation indicated that the structural changes were partly a consequence of membrane unstacking. The analysis of both an LHCSR loss-offunction mutant, $n p q 4$ lhcsr 1, and a regulatory mutant that over-expresses LHCSR, spal-1, showed that structural changes occurred independently of LHCSR protein levels, demonstrating that LHCSR was neither necessary nor sufficient to induce the thylakoid structural changes associated with HL acclimation. In contrast, stt7-9, a mutant lacking a kinase of major light-harvesting antenna proteins, had a distinct thylakoid structural response during HL acclimation relative to all other lines tested. Thus, while LHCSR and the antenna protein phosphorylation are core features of HL acclimation, it appears that only the latter acts as a determinant for thylakoid structural rearrangements. These results indicate that two independent mechanisms occur simultaneously to cope with HL conditions. Possible scenarios for HL-induced thylakoid structural changes are discussed.
\end{abstract}




\section{INTRODUCTION}

Understanding natural photosynthetic systems is crucial for developing efficient technologies for bioenergy. In green algae and plants, photosynthetic electron transport is initiated by the absorption of light energy mainly by light-harvesting complex (LHC) proteins in chloroplast thylakoid membranes (Jansson, 1999, Wobbe et al., 2016). Light energy is essential for photosynthesis, but an excess amount of absorbed energy can cause photooxidative damage, which depresses photosynthetic efficiency and growth (Aro et al., 1993, Li et al., 2009, Pinnola and Bassi, 2018). Therefore, photosynthetic organisms have evolved photoprotective mechanisms. One of the most important mechanisms is nonphotochemical quenching (NPQ) of chlorophyll (Chl) fluorescence, which harmlessly dissipates excess excitation energy as heat (Muller et al., 2001, Ruban, 2016). The fast-acting component of NPQ, qE, is tightly regulated by a proton gradient across the thylakoid membrane $(\Delta \mathrm{pH})$. The $\Delta \mathrm{pH}$ is determined by the rate of water oxidation catalyzed by the oxygen-evolving complex of photosystem II (PSII) and the Q-cycle of the cytochrome $b_{6} f$ complex in the light (Kramer et al., 2004). This light-induced increase in $\Delta \mathrm{pH}$ is essential for not only generation of ATP by ATP synthase but also the feedback induction of $\mathrm{qE}$ to minimize photooxidative damage in thylakoid membranes under fluctuating light conditions in natural environments.

An increased flow of photosynthetic electrons from PSII also causes the accumulation of reduced plastoquinone (PQ) pool in thylakoid membranes, which triggers another component of NPQ, qT or state transitions. The reduced PQ pool activates the Stt7/STN7 kinase, which phosphorylates LHC proteins of PSII (LHCII) to induce redistribution of excitation energy from PSII to photosystem I (PSI) via increased connectivity between LHCII and PSI (Minagawa, 2011, Rochaix, 2011, Goldschmidt-Clermont and Bassi, 2015). The redistribution of excitation energy between the two photosystems prevents excessive excitation of PSII, while PSI converts excitation energy more efficiently (Trissl and Wilhelm, 1993). The time scale of activation of state transitions is relatively longer than $\mathrm{qE}$, because it involves dynamic protein reorganization within thylakoid membranes.

NPQ is ubiquitous among oxygenic photosynthetic organisms, although the molecular processes are different (Niyogi and Truong, 2013, Pinnola, 2019). In the model unicellular green alga Chlamydomonas reinhardtii, the stress-related LHC proteins, called LHCSRs, are essential for the qE component of NPQ (Peers et al., 2009). Similar to components of the major LHC antenna system, LHCSR is a membrane protein with three transmembrane helices and contains Chls and carotenoids (Bonente et al., 2011, Liguori et al., 2013). The $\mathrm{qE}$ facilitated by LHCSR is regulated by the protonation state of acidic residues on the lumenal side of the protein, and this protonation state responds to changes in $\Delta \mathrm{pH}$ under high light (HL) conditions (Ballottari et al., 2016, Dinc et al., 2016, Troiano et al., 2021). Intriguingly, LHCSR protein level in thylakoid membranes is very limited under low light (LL) conditions (Petroutsos et al., 2011, Maruyama et al., 2014). Recently, it has been shown that the expression of NPQ-related genes, including 
LHCSRs, is negatively regulated by a conserved E3 ubiquitin ligase complex, which targets the transcriptional activators for $\mathrm{qE}$ gene expression in LL for proteolysis (Aihara et al., 2019, Gabilly et al., 2019, Tokutsu et al., 2019). Under HL conditions, the E3 ubiquitin ligase activity is inactivated by a photoreceptor-dependent mechanism, which allows the transcriptional activation of the qE-related genes. The inhibition of the E3 ubiquitin ligase activity by a mutation in its component SPA1 results in the accumulation of LHCSR proteins even under LL conditions (Gabilly et al., 2019, Tokutsu et al., 2019). Thus, the induction of high NPQ activity in $C$. reinhardtii is tightly regulated during HL acclimation by both transcriptional and post-translational control of LHCSR activity in thylakoid membranes.

The molecular genetics and biochemistry of HL acclimation mechanisms in C. reinhardtii have been extensively studied (Allorent and Petroutsos, 2017, Rochaix and Bassi, 2019). Yet, the dynamics of the thylakoid membrane macrostructural response to HL acclimation have not been explored. In this study, we combined subdiffraction-resolution microscopy and analytical membrane subfractionation to investigate the macroscale structural changes of thylakoid architecture during HL acclimation in C. reinhardtii. We observed a drastic change in thylakoid structure during HL acclimation by using subdiffraction-resolution imaging analysis. We also observed changes in the densities of isolated thylakoid membranes, which were analytically separated by density-dependent fractionation. To investigate a correlation between the structural changes of thylakoid membranes and qE capacity, we used the npq4 lhcsrl mutant lacking LHCSR proteins (Ballottari et al., 2016) and the spal-1 mutant (Gabilly et al., 2019), in which LHCSR proteins are accumulated under LL. Regardless of the accumulation of LHCSR proteins, both mutants exhibited similar structural changes to wild-type (WT) after HL acclimation, demonstrating that the HLinduced changes in thylakoid structures occur independently of LHCSR function. On the other hand, the stt7-9 mutant, which is deficient in state transitions, did not undergo the macroscale structural changes that occur in WT after HL acclimation. The two combined technical approaches revealed that the aspects of the macroscale membrane architecture and the membrane stacking can be characterized separately.

\section{RESULTS}

To observe thylakoid membranes in C. reinhardtii, we used a confocal microscope with Airyscan, which is an array of $32 \mathrm{GaAsP}$ photomultiplier tube detectors arranged in a hexagonal pattern and is capable of subdiffraction-resolution imaging through determining the microscope point spread function that is projected in the center of the array detectors (Huff et al., 2017). The spatial resolution achievable by Airyscan microscopy is theoretically lower than that of structured illumination microscopy (SIM). However, it is reported that Airyscan microscopy performs better than SIM for samples with a lower signal-to-noise ratio (Sivaguru et al., 2018). As compared to our previous study using SIM (Iwai et al., 2018), Airyscan images contained no obvious artifact due to lower signal-to-noise ratios. Therefore, these two microscopes 
are complementary techniques to obtain subdiffraction resolution.

We first observed live $C$. reinhardtii cells acclimated to LL $\left(\sim 30 \mu \mathrm{mol}\right.$ photons $\left.\mathrm{m}^{-2} \mathrm{~s}^{-1}\right)$ using the Airyscan microscope. The Airyscan images showed typical thylakoid structures in the cup-shaped chloroplast very similar to the ones as previously observed using SIM (Iwai et al., 2018). The LLacclimated cells typically showed undisturbed thylakoid layers with relatively little space between the layers throughout the lobe regions (Figure 1a, b). By optical sectioning through the z-axis, it was possible to observe the membrane surface patterns (Figure 1c), which are usually difficult to obtain by electron microscopy (EM) observation. The Airyscan image revealed smooth fluorescence patterns indicating continuous membrane regions with occasional empty spaces. Layers of thylakoid membranes in the lobe and thylakoid tubules were also visible (Figure 1d, e). On the other hand, after HL $\left(\sim 350 \mu \mathrm{mol}\right.$ photons $\mathrm{m}^{-}$ ${ }^{2} \mathrm{~s}^{-1}$ ) acclimation for $24 \mathrm{~h}$, the overall thylakoid structures appeared to be thinner than that of the LLacclimated cells. The thylakoid structures at the lobe showed fewer layers with more non-fluorescent spaces between the layers (Figure 2a-d). Similarly, the thylakoid structures at the base became thinned, and the space occupied by the pyrenoid became larger than that of the LL-acclimated cells. Optical sectioning showed that the surface pattern became rough and less dense due to the thinning of membranes (Figure 2e). Further, the lobe regions appeared to be shorter and sometimes partially disappeared.

Similar characteristics were observed under a transmission EM (TEM). The TEM images of the LLacclimated cells revealed that thylakoid membranes were continuously undisturbed with narrow spaces between the membranes (Figure 3a). The membranes at the lobe regions were close to each other, and the ones at the base were also tightly associated near the pyrenoid (Figure 3b, c). The TEM images of the HLacclimated cells clearly showed more stromal spaces between the membranes throughout the thylakoid structures (Figure 3d). In the lobe regions, there were larger spaces between the membranes, but also some membranes appeared to be associated tightly with each other (Figure 3e, g, h). Interestingly, the enlarged space for the pyrenoid is actually the stromal space around the pyrenoid, as the size of the pyrenoid did not significantly change under HL (Figure 3f). The membranes in the base regions also appeared more spaced out (Figure 3f, i). These TEM results demonstrated consistency with the Airyscan images of the thinner thylakoid structures and more space between membranes observed in the HL-acclimated cells.

To gain more insight into the thylakoid membrane structures, we performed analytical densitydependent fractionation of isolated thylakoid membranes using sucrose step-wise gradient centrifugation. This experiment showed that the chloroplasts in the LL-acclimated cells contained a thick green band of the densest membrane fraction (T4) and a less distinct green band of a lighter membrane fraction (T3) (Figure 4a). In contrast, the chloroplasts in the HL-acclimated cells contained four green bands - the T4 fraction became least abundant, while the second lighter fraction (T2) and T3 fractions became abundant, and the lightest fraction (T1) became visible (Figure 4a). An increase of carotenoid was also visible above 
the $\mathrm{T} 1$ fraction. The increase in quantity of the lighter membrane fractions in the HL-acclimated cells is consistent with the imaging results that showed that overall thylakoid structures became thinned in the HLacclimated cells (Figure 2). Surprisingly, an increase in T1-T3 fractions was already generated after 15 min of HL treatment (Figure 4b). A gradual increase in T1 and T2 was observed from 15 min to $1 \mathrm{~h}$ of HL treatment, while the accumulation of T4 declined. The membrane heights measured using atomic force microscopy (AFM) also indicated that the T4 fraction contained thicker membranes than T3, and the membranes in $\mathrm{T} 1+\mathrm{T} 2$ fractions were thinnest (Figure 4c). The changes in height reflects the number of membrane stacks, as thylakoid membranes in $C$. reinhardtii also form the appressed and non-appressed membrane regions. Recently, the lateral heterogeneity of the two photosystems has been shown by cryoelectron tomography; PSII is located in the appressed regions, while PSI is physically segregated into the non-appressed regions (Wietrzynski et al., 2020). Chl fluorescence spectra at $77 \mathrm{~K}$ revealed that the T4 from LL contained the emission peaks for both PSII and PSI, but the T3 from HL showed much less PSI emission than the T1+T2 from HL (Figure 4d). These results suggest that the HL-induced thylakoid structural changes involve membrane unstacking.

HL induces both $\triangle \mathrm{pH}$ and LHCSR expression, which are essential components for NPQ induction in C. reinhardtii. Given its central role in NPQ and high light acclimation, one hypothesized role of LHCSR has been the induction of thylakoid structural changes during HL acclimation. We tested this directly by using the npq4 lhcsrl mutant, which lacks LHCSR proteins and has low NPQ capacity even after HL acclimation (Ballottari et al., 2016). Airyscan image analysis revealed that the structural changes of thylakoid membranes in $n p q 4$ lhcsr 1 were very similar to that of WT after HL acclimation - thinned lobe structures, more space between membranes, and a larger stromal space around the pyrenoid (Figure 5a-d). Analytical density-dependent fractionation of isolated thylakoid membranes also showed similar resultsan increase in T1, T2, and T3 fractions and a decrease in T4 after HL acclimation (Figure 5e). These results indicate that the LHCSRs are not necessary for the thylakoid structural changes during HL acclimation. Additionally, the low NPQ capacity of HL-grown npq4 lhcsrl demonstrates that thylakoid membrane structural changes are not sufficient to induce $\mathrm{qE}$ in the absence of LHCSRs in C. reinhardtii.

The results using npq4 lhcsrl suggest that thylakoid structural changes do not directly influence the $\mathrm{qE}$ capacity. To test this further, we used the spal-1 mutant, in which LHCSR accumulates at high levels even under LL (Gabilly et al., 2019). While the stromal space around the pyrenoid was already enlarged before HL acclimation in spal-1 (Figure 5f, g), TEM images of LL-grown spal-1 cells revealed that the enlarged stromal space around the pyrenoid was likely a consequence of high starch accumulation in the pyrenoid, which was not observed in WT (Figure S1). After HL acclimation, similar structural changes were observed in spal-1 relative to WT (Figure 5h, i). Analytical density-dependent fractionation analysis still showed results similar to WT-more T4 fraction in LL and an increase in T1, T2, and T3 fractions after HL 
acclimation (Figure 5j). Taken together, these results reveal that LHCSRs in thylakoid membranes are not sufficient to cause the membrane unstacking observed during HL acclimation. The fact that LL-grown spal1 has a high NPQ capacity (Gabilly et al., 2019), albeit at reduced levels relative to HL-acclimated cells, also demonstrates that LHCSR-mediated induction of NPQ can at least partly occur in the absence of the thylakoid membrane structural rearrangement associated with HL acclimation. Therefore, LHCSRs are neither necessary nor sufficient to induce the thylakoid membrane structural rearrangement associated with HL acclimation, and LHCSR-mediated NPQ can operate, at least partly, in the absence of these structural rearrangements.

An alternative consideration to an LHCSR-dependent signal for thylakoid membrane structural rearrangements in HL is that they might relate to photosynthetic electron transport itself. To investigate whether the HL-induced thylakoid structural changes are dependent on photosynthetic electron transport, we treated $C$. reinhardtii cells with 3-(3,4-dichlorophenyl)-1,1-dimethylurea (DCMU), which blocks electron transfer from PSII to PQ, inhibiting photosynthetic linear electron transport. Interestingly, the results demonstrated that the accumulation of $\mathrm{T} 1$ and $\mathrm{T} 2$ fractions was suppressed by DCMU treatment, and accumulation of the T4 fraction increased relative to samples without DCMU (Figure 6a). These samples were harvested after $15 \mathrm{~min}$ of HL to limit the severe photodamage that HL induces in DCMUtreated cells. However, 15 min was sufficient to induce thylakoid structural changes similar to that observed after $24 \mathrm{~h}$ HL treatment in untreated cells (Fig. 4a-b).

PQ remains oxidized upon DCMU treatment. The PQ redox state is known to affect state transitions in C. reinhardtii, which maintains the energy balance between PSI and PSII by dynamically redistributing excitation energy absorption between the two photosystems via reorganization of LHCII (Minagawa, 2011, Rochaix, 2011, Goldschmidt-Clermont and Bassi, 2015). DCMU-treatment locks cells in the so-called "state 1 conditions" in which LHCII remains unphosphorylated (Allen et al., 1981), and excitation energy is preferentially transferred to PSII (Finazzi et al., 2002, Iwai et al., 2008).

To test whether the HL-induced structural changes are related to state transitions, we used the stt7-9 mutant, which lacks the Stt7 kinase that phosphorylates LHCII (Depege et al., 2003) and thus does not perform state transitions. Intriguingly, there were less pronounced structural changes between LL- and HLacclimated stt7-9 cells than that observed in WT (Figure 6b-e). This result is in agreement with the observation in Arabidopsis, in which LHCII phosphorylation causes dynamic thylakoid membrane stacking (Fristedt et al., 2009, Wood et al., 2019). However, analytical density-dependent fractionation analysis still showed similar results to WT (Figure 6f). Because the state transition is a short-term light acclimation mechanism, HL treatment for $24 \mathrm{~h}$ might cause other long-term acclimation mechanisms. To observe the short-term effect on thylakoid structural changes, we treated both WT and the stt7-9 mutant under HL for $1 \mathrm{~h}$ and analyzed them by density-dependent fractionation analysis. Interestingly, the result showed that the 
stt7-9 cells had much less T1 and T2 and more T3 and T4 than in the WT (Figure 6g). Thus, at least for a short-term response to HL, LHCII phosphorylation is necessary for the membrane unstacking as observed in WT. These results indicate that Airyscan imaging and analytical density-dependent fractionation actually characterize aspects of thylakoid membrane structures that are at different spatial levels - the former visualizes overall membrane structural shapes, while the latter specifically distinguishes the level of membrane stacking.

\section{DISCUSSION}

Our observations demonstrated that macroscale structural changes of thylakoid membranes occur in $C$. reinhardtii during HL acclimation (Figures 1-4). According to the results using $n p q 4$ lhcsr 1 and spa1-1, the HL-induced structural changes are not directly induced by the accumulation of LHCSR proteins in thylakoid membranes (Figure 5). These results also reconfirmed that high NPQ capacity in $C$. reinhardtii is strongly correlated with the LHCSR protein level in thylakoid membranes but not with the macroscale structural changes of thylakoid membranes. Notably, the NPQ capacity of spa1-1, which accumulates high LHCSR proteins even under LL, was higher in LL but still increased further after HL acclimation (Gabilly et al., 2019). Thus, while LHCSR is neither necessary nor sufficient to induce thylakoid structural rearrangements, the high NPQ capacity of HL-acclimated cells may well depend on the thylakoid structural changes during HL acclimation.

DCMU not only blocks linear electron transport but also the HL-induced expression of LHCSRs (Petroutsos et al., 2011, Maruyama et al., 2014). Interestingly, the DCMU-treated cells did not show the structural changes under HL, at least during a short period of time of HL exposure (Figure 6a). The reduced state of the PQ pool also induces LHCII phosphorylation (Finazzi, 2005, Rochaix, 2007). Although the lack of LHCII phosphorylation in state transitions does not directly affect NPQ capacity (Girolomoni et al., 2019), it has been shown that LHCSR can move to PSI during state transitions (Allorent et al., 2013). We observed that the lack of LHCII phosphorylation indeed prevented membrane unstacking in the beginning of HL treatment (Figure $6 \mathrm{~g}$ ). Thus, it seems likely that thylakoid membrane protein reorganization in a short-term HL acclimation depends on LHCII phosphorylation. However, prolonged HL conditions (e.g. $24 \mathrm{~h}$ ) eventually generate a similar level of unstacked membranes to that of WT in the stt7-9 mutant (Figure 6f). Unexpectedly, Airyscan images did not show the pronounced HL phenotypes in the stt7-9 mutant after $24 \mathrm{~h}$ of HL treatment (Figure 6d, e), suggesting that the macroscale membrane architecture and the level of membrane stacking need to be determined separately.

Another possible relationship worth considering is the thylakoid membrane structures and how they facilitate and influence protein assembly and insertion into thylakoid membranes. During HL acclimation, the newly translated LHCSR proteins need to be inserted in a way that excitation energy is efficiently 
transferred from LHCII to be dissipated by LHCSR. The base region of the C. reinhardtii chloroplast near the pyrenoid is known as the translation zone, in which protein translation and assembly as well as Chl biosynthesis occur (Schottkowski et al., 2012, Sun et al., 2019). Our imaging results showed thinning of the overall thylakoid structures, including the translation zone, and the enlarged stromal space at the base region during HL acclimation (Figs. 2, 3). To be functional, newly synthesized LHCSR proteins need to be assembled with Chls and carotenoids (Bonente et al., 2011). The HL-induced structural changes of thylakoid membranes might be coordinated with protein assembly and insertion mechanisms, and the membrane unstacking may enable newly synthesized photoprotective proteins such as LHCSR to have access to membranes to be inserted. This would provide a mechanistic explanation for why ectopic expression of LHCSR in LL is not sufficient to induce levels of NPQ equal to HL-grown cells (Gabilly et al., 2019).

It is worth mentioning the limitations of imaging analysis by observing Chl fluorescence. It is practical to assume that the structures based on $\mathrm{Chl}$ fluorescence imaging reflect thylakoid membranes because the membranes are filled with Chl pigments. However, Chl fluorescence emission from PSI is difficult to observe at room temperature, especially with confocal microscopes, due to its higher excitation trapping than PSII (Trissl and Wilhelm, 1993). Also, fluorescence intensity frequently fluctuates due to several factors, including NPQ and photosynthetic electron transport, that can influence the interpretation of observed structural differences in thylakoid membranes due to different intensities of Chl fluorescence between LL- and HL-acclimated cells. Therefore, it is necessary to use TEM to confirm the structural changes in thylakoid membranes observed by Airyscan microscopy (Figure 3). The TEM images also reveal the similar structural differences in thylakoid membranes and indicate that Airyscan microscopy is capable of visualizing the overall thylakoid structures sufficiently to reveal the differences between the LL- and HL-acclimated cells. It should be noted that, although large stromal spaces between membranes are more visible in the HL-acclimated cells than that of the LL-acclimated cells, it also appears that a lot of membranes in the HL-acclimated cells are associated closely with each other (Figure 3e, g, h). This might suggest a possible alteration of refractive indices of the membrane, which would affect how much light is absorbed by LHC proteins (Capretti et al., 2019). However, because of the chemical fixation and negative staining done in conventional TEM, it is difficult to determine whether changes in such membrane compartmental spaces are correlated with native conditions. Although bioimaging techniques provide images at lower resolution than do EM techniques, it is feasible to evaluate a large number of samples, which is inherently practical to determine statistically supported characteristics when examined under EM. Therefore, our observation will be helpful when microscopic details of the thylakoid compartment, stacking, and protein organization will be examined by advanced cryo-EM techniques. 


\section{EXPERIMENTAL PROCEDURES}

\section{Strains, growth conditions, and HL treatment}

C. reinhardtii WT strain 4A+ (mt+, 137c background; CC-4051), npq4 lhcsrl, and spal-1, and stt7-9 mutants were grown in Tris-acetate-phosphate liquid media (Harris et al., 1989) as described previously (Niyogi et al., 1997). The culture was adjusted to $\sim 1 \mu \mathrm{g} \mathrm{Chl} / \mathrm{mL}$ and incubated on a shaker under $\sim 30 \mu \mathrm{mol}$ photons $\mathrm{m}^{-2} \mathrm{~s}^{-1}(\mathrm{LL})$ at $25^{\circ} \mathrm{C}$ for $3 \mathrm{~d}$. For HL treatment, the LL-acclimated cells were diluted to $1 \mu \mathrm{g} \mathrm{Chl} / \mathrm{mL}$ (for $24 \mathrm{~h}$ treatment) or $2 \mu \mathrm{g} \mathrm{Chl} / \mathrm{mL}$ (for $15 \mathrm{~min}$ and $1 \mathrm{~h}$ treatments) in fresh TAP liquid media and incubated under $\sim 350 \mu \mathrm{mol}$ photons $\mathrm{m}^{-2} \mathrm{~s}^{-1}$ at $25^{\circ} \mathrm{C}$ for specified duration of time. For the 24 -h incubation under HL, the culture was diluted again after $6 \mathrm{~h}$ of HL incubation to maintain a low cell density. Chl concentration was measured as described previously (Porra et al., 1989).

\section{Airyscan microscopy}

C. reinhardtii cells were observed using a Zeiss LSM 880 microscope equipped with the Airyscan detector with a Zeiss Plan-Apochromat 63×/1.4 NA DIC M27 Oil objective. Chls were excited with $633 \mathrm{~nm}$ laser, and fluorescence was acquired through a $645 \mathrm{~nm}$ longpass filter. Image acquisition and analysis were done under the full control of ZEN software (Zeiss) and ImageJ software (US National Institutes of Health, https://www.nih.gov/).

\section{TEM}

C. reinhardtii cells acclimated under LL and HL $24 \mathrm{~h}$ were centrifuged, resuspended, and fixed with 2\% glutaraldehyde in TAP liquid media for $24 \mathrm{~h}$ at $4{ }^{\circ} \mathrm{C}$ in the dark. The fixed cells were washed and post-fixed with a sodium cacodylate buffer containing $1 \%(\mathrm{w} / \mathrm{v})$ osmium tetroxide and $0.8 \%(\mathrm{w} / \mathrm{v})$ potassium ferricyanide for $2 \mathrm{~h}$. The fixed cells were rinsed with cacodylate buffer for 10 min twice. The fixed cells were dehydrated with increasing concentrations of acetone (35-100\%). The dehydrated cells were then infiltrated and embedded in resin. Sections were cut to approximately $500 \mu \mathrm{m}$ in diameter and $60 \mathrm{~nm}$ in thickness. The thin sections were collected on Maxtaform copper slot grids $(2 \times 1-\mathrm{mm}$ oval hole $)$ that had been coated with $0.5 \%$ formvar. Sections were dried and post-stained with $2 \%$ uranyl acetate for $7 \mathrm{~min}$, followed by lead citrate for $7 \mathrm{~min}$. The sections were dried and examined using a JEOL $1200 \mathrm{EX}$ transmission electron microscope at $100 \mathrm{kV}$.

\section{AFM}

Height of isolated thylakoid membrane was measured as described previously (Iwai et al., 2013). Briefly, a freshly cleaved mica surface was treated with adsorption buffer $(10 \mathrm{mM}$ Tris- $\mathrm{HCl}(\mathrm{pH} 7.3), 150 \mathrm{mM} \mathrm{KCl}$, $20 \mathrm{mM} \mathrm{MgCl} 2)$. Then, isolated thylakoid membranes $(0.25 \mu \mathrm{g} \mathrm{Chl} / \mu \mathrm{L})$ were added to the adsorption buffer 
on the mica surface and incubated for $10 \mathrm{~min}$. After gentle wash with MilliQ water, the membranes were observed by using a commercial MFP-3D stand-alone AFM in tapping mode in air (Asylum Research). Silicon cantilevers with a length of $240 \mu \mathrm{m}(k=2 \mathrm{~N} / \mathrm{m}$; OMCL-AC240TS-C2, Olympus) were used. Averaged height was calculated from the membrane areas of at least $50 \mu \mathrm{m}^{2}$.

\section{Analytical sucrose density-dependent membrane fractionation}

C. reinhardtii cells were collected at $1,870 \times g$ and $4^{\circ} \mathrm{C}$ for $5 \min$ (JLA9.1000, Beckman Coulter). The cells were resuspended at $0.125 \mathrm{mg} \mathrm{Chl} / \mathrm{mL}$ with disruption buffer containing $25 \mathrm{mM}$ MES-NaOH (pH 6.5), $0.33 \mathrm{M}$ sucrose, $1.5 \mathrm{mM} \mathrm{NaCl}, 0.2 \mathrm{mM}$ benzamidine, and $1 \mathrm{mM} \varepsilon$-aminocaproic acid at $4^{\circ} \mathrm{C}$. The cells were disrupted at $5 \mathrm{kpsi}$ once using the MC Cell Disruptor (Constant Systems Ltd, Northants, UK). The disrupted cells ( $0.5 \mathrm{mg} \mathrm{Chl} \mathrm{in} 4 \mathrm{~mL}$ ) were loaded onto sucrose step-wise gradient, containing $0.9,1.2,1.5$, and $1.8 \mathrm{M}$ sucrose with $25 \mathrm{mM}$ MES-NaOH (pH 6.5) and $1.5 \mathrm{mM} \mathrm{NaCl}$ ( $2 \mathrm{~mL} /$ each sucrose layer). The thylakoid membranes with different densities were analytically separated at $125,000 \times g$ and $4^{\circ} \mathrm{C}$ for $1 \mathrm{~h}(\mathrm{SW} 41 \mathrm{Ti}$, Beckman Coulter).

\section{Fluorescence emission spectroscopy at $77 \mathrm{~K}$}

The sample obtained by analytical sucrose density-dependent membrane fractionation was placed in a glass tube and frozen in liquid nitrogen. Fluorescence emission was recorded at $77 \mathrm{~K}$ using FluoroMax-4 spectrophotometer (Horiba Scientific). Excitation wavelength was $440 \mathrm{~nm}$ with a 2-nm slit size. Emission wavelength measured was from 650 to $800 \mathrm{~nm}$ with a 2-nm slit size. Fluorescence emission for each sample was recorded consecutively three times to obtain averaged spectra.

\section{ACKNOWLEDGEMENTS}

We thank Christopher R. Baker, Setsuko Wakao, and Valle Ojeda for their critical reading of the manuscript; Holly Aaron and Feather Ives at the Molecular Imaging Center at University of California, Berkeley for the technical setup for Airyscan microscopy; and Reena Zalpuri at the Electron Microscope Laboratory at University of California, Berkeley for assistance in EM sample preparation and data collection. This work was supported by the U.S. Department of Energy, Office of Science, through the Photosynthetic Systems program in the Office of Basic Energy Sciences. K.K.N. is an investigator of the Howard Hughes Medical Institute.

\section{CONFLICT OF INTEREST}

The authors declare no conflict of interest. 


\section{SUPPORTING INFORMATION}

Figure S1. The representative TEM image of the $C$. reinhardtii spal-1 cell acclimated to LL conditions.

\section{REFERENCES}

Aihara, Y., Fujimura-Kamada, K., Yamasaki, T. and Minagawa, J. (2019) Algal photoprotection is regulated by the E3 ligase CUL4-DDB1(DET1). Nat. Plants, 5, 34-40.

Allen, J.F., Bennet, J., Steinback, K.E. and Arntzen, C.J. (1981) Chloroplast protein phosphorylation couples plastoquinone redox state to distribution of excitation energy between photosystems. Nature, 291, 21-25.

Allorent, G. and Petroutsos, D. (2017) Photoreceptor-dependent regulation of photoprotection. Curr. Opin. Plant Biol., $37,102-108$.

Allorent, G., Tokutsu, R., Roach, T., Peers, G., Cardol, P., Girard-Bascou, J., Seigneurin-Berny, D., Petroutsos, D., Kuntz, M., Breyton, C., Franck, F., Wollman, F.A., Niyogi, K.K., Krieger-Liszkay, A., Minagawa, J. and Finazzi, G. (2013) A dual strategy to cope with high light in Chlamydomonas reinhardtii. Plant Cell, 25, 545557.

Aro, E.M., Virgin, I. and Andersson, B. (1993) Photoinhibition of photosystem II. Inactivation, protein damage and turnover. Biochim. Biophys. Acta, 1143, 113-134.

Ballottari, M., Truong, T.B., De Re, E., Erickson, E., Stella, G.R., Fleming, G.R., Bassi, R. and Niyogi, K.K. (2016) Identification of $\mathrm{pH}$-sensing sites in the light harvesting complex stress-related 3 protein essential for triggering non-photochemical quenching in Chlamydomonas reinhardtii. J. Biol. Chem., 291, 7334-7346.

Bonente, G., Ballottari, M., Truong, T.B., Morosinotto, T., Ahn, T.K., Fleming, G.R., Niyogi, K.K. and Bassi, R. (2011) Analysis of LhcSR3, a protein essential for feedback de-excitation in the green alga Chlamydomonas reinhardtii. PLoS Biol., 9, e1000577.

Capretti, A., Ringsmuth, A.K., van Velzen, J.F., Rosnik, A., Croce, R. and Gregorkiewicz, T. (2019) Nanophotonics of higher-plant photosynthetic membranes. Light Sci. Appl., 8, 5.

Depege, N., Bellafiore, S. and Rochaix, J.D. (2003) Role of chloroplast protein kinase Stt7 in LHCII phosphorylation and state transition in Chlamydomonas. Science, 299, 1572-1575.

Dinc, E., Tian, L., Roy, L.M., Roth, R., Goodenough, U. and Croce, R. (2016) LHCSR1 induces a fast and reversible $\mathrm{pH}-$ dependent fluorescence quenching in LHCII in Chlamydomonas reinhardtii cells. Proc. Natl Acad. Sci. USA, 113, 7673-7678.

Engel, B.D., Schaffer, M., Kuhn Cuellar, L., Villa, E., Plitzko, J.M. and Baumeister, W. (2015) Native architecture of the Chlamydomonas chloroplast revealed by in situ cryo-electron tomography. eLife, 4, e04889.

Finazzi, G. (2005) The central role of the green alga Chlamydomonas reinhardtii in revealing the mechanism of state transitions. J. Exp. Bot., 56, 383-388.

Finazzi, G., Rappaport, F., Furia, A., Fleischmann, M., Rochaix, J.D., Zito, F. and Forti, G. (2002) Involvement of state transitions in the switch between linear and cyclic electron flow in Chlamydomonas reinhardtii. EMBO Rep., 3, 280-285.

Fristedt, R., Willig, A., Granath, P., Crevecoeur, M., Rochaix, J.D. and Vener, A.V. (2009) Phosphorylation of photosystem II controls functional macroscopic folding of photosynthetic membranes in Arabidopsis. Plant Cell, 21, 3950-3964.

Gabilly, S.T., Baker, C.R., Wakao, S., Crisanto, T., Guan, K., Bi, K., Guiet, E., Guadagno, C.R. and Niyogi, K.K. (2019) Regulation of photoprotection gene expression in Chlamydomonas by a putative E3 ubiquitin ligase complex and a homolog of CONSTANS. Proc. Natl Acad. Sci. USA, 116, 17556-17562.

Girolomoni, L., Cazzaniga, S., Pinnola, A., Perozeni, F., Ballottari, M. and Bassi, R. (2019) LHCSR3 is a nonphotochemical quencher of both photosystems in Chlamydomonas reinhardtii. Proc. Natl Acad. Sci. USA, 201809812. 
Goldschmidt-Clermont, M. and Bassi, R. (2015) Sharing light between two photosystems: mechanism of state transitions. Curr. Opin. Plant Biol., 25, 71-78.

Harris, E.H., Stern, D.B. and Witman, G.B. (1989) The Chlamydomonas Sourcebook: Academic Press San Diego.

Huff, J., Bergter, A., Birkenbeil, J., Kleppe, I., Engelmann, R. and Krzic, U. (2017) The new 2D Superresolution mode for ZEISS Airyscan. Nat. Methods, 14, 1223-1223.

Iwai, M., Pack, C.G., Takenaka, Y., Sako, Y. and Nakano, A. (2013) Photosystem II antenna phosphorylationdependent protein diffusion determined by fluorescence correlation spectroscopy. Sci. Rep., 3, 2833.

Iwai, M., Roth, M.S. and Niyogi, K.K. (2018) Subdiffraction-resolution live-cell imaging for visualizing thylakoid membranes. Plant J., 96, 233-243.

Iwai, M., Takahashi, Y. and Minagawa, J. (2008) Molecular remodeling of photosystem II during state transitions in Chlamydomonas reinhardtii. Plant Cell, 20, 2177-2189.

Jansson, S. (1999) A guide to the Lhc genes and their relatives in Arabidopsis. Trends Plant Sci., 4, 236-240.

Kramer, D.M., Avenson, T.J. and Edwards, G.E. (2004) Dynamic flexibility in the light reactions of photosynthesis governed by both electron and proton transfer reactions. Trends Plant Sci., 9, 349-357.

Li, Z., Wakao, S., Fischer, B.B. and Niyogi, K.K. (2009) Sensing and responding to excess light. Annu. Rev. Plant Biol., 60, 239-260.

Liguori, N., Roy, L.M., Opacic, M., Durand, G. and Croce, R. (2013) Regulation of light harvesting in the green alga Chlamydomonas reinhardtii: the C-terminus of LHCSR is the knob of a dimmer switch. J. Am. Chem. Soc., $135,18339-18342$.

Maruyama, S., Tokutsu, R. and Minagawa, J. (2014) Transcriptional regulation of the stress-responsive light harvesting complex genes in Chlamydomonas reinhardtii. Plant Cell Physiol, 55, 1304-1310.

Minagawa, J. (2011) State transitions-The molecular remodeling of photosynthetic supercomplexes that controls energy flow in the chloroplast. Biochim. Biophys. Acta, 1807, 897-905.

Muller, P., Li, X.P. and Niyogi, K.K. (2001) Non-photochemical quenching. A response to excess light energy. Plant Physiol., 125, 1558-1566.

Niyogi, K.K., Bjorkman, O. and Grossman, A.R. (1997) Chlamydomonas xanthophyll cycle mutants identified by video imaging of chlorophyll fluorescence quenching. Plant Cell, 9, 1369-1380.

Niyogi, K.K. and Truong, T.B. (2013) Evolution of flexible non-photochemical quenching mechanisms that regulate light harvesting in oxygenic photosynthesis. Curr. Opin. Plant Biol., 16, 307-314.

Peers, G., Truong, T.B., Ostendorf, E., Busch, A., Elrad, D., Grossman, A.R., Hippler, M. and Niyogi, K.K. (2009) An ancient light-harvesting protein is critical for the regulation of algal photosynthesis. Nature, 462, 518-521.

Petroutsos, D., Busch, A., Janssen, I., Trompelt, K., Bergner, S.V., Weinl, S., Holtkamp, M., Karst, U., Kudla, J. and Hippler, M. (2011) The chloroplast calcium sensor CAS is required for photoacclimation in Chlamydomonas reinhardtii. Plant Cell, 23, 2950-2963.

Pinnola, A. (2019) The rise and fall of Light-Harvesting Complex Stress-Related proteins as photoprotection agents during evolution. J. Exp. Bot., 70, 5527-5535.

Pinnola, A. and Bassi, R. (2018) Molecular mechanisms involved in plant photoprotection. Biochem. Soc. Trans., 46, 467-482.

Porra, R.J., Thompson, W.A. and Kriedemann, P.E. (1989) Determination of accurate extinction coefficients and simultaneous equations for assaying chlorophylls $a$ and $b$ extracted with four different solvents: verification of the concentration of chlorophyll standards by atomic absorption spectroscopy. Biochim. Biophys. Acta, 975, 384-394.

Rochaix, J.-D. and Bassi, R. (2019) LHC-like proteins involved in stress responses and biogenesis/repair of the photosynthetic apparatus. Biochem. J., 476, 581-593.

Rochaix, J.D. (2007) Role of thylakoid protein kinases in photosynthetic acclimation. FEBS Lett., 581, 2768-2775. 
Rochaix, J.D. (2011) Regulation of photosynthetic electron transport. Biochim. Biophys. Acta, 1807, 375-383.

Ruban, A.V. (2016) Nonphotochemical chlorophyll fluorescence quenching: Mechanism and effectiveness in protecting plants from photodamage. Plant Physiol., 170, 1903-1916.

Schottkowski, M., Peters, M., Zhan, Y., Rifai, O., Zhang, Y. and Zerges, W. (2012) Biogenic membranes of the chloroplast in Chlamydomonas reinhardtii. Proc. Natl Acad. Sci. USA, 109, 19286-19291.

Sivaguru, M., Urban, M.A., Fried, G., Wesseln, C.J., Mander, L. and Punyasena, S.W. (2018) Comparative performance of airyscan and structured illumination superresolution microscopy in the study of the surface texture and 3D shape of pollen. Microsc. Res. Tech., 81, 101-114.

Sun, Y., Valente-Paterno, M.I., Bakhtiari, S., Law, C., Zhan, Y. and Zerges, W. (2019) Photosystem biogenesis is localized to the translation zone in the chloroplast of Chlamydomonas. Plant Cell, 31, 3057-3072.

Tokutsu, R., Fujimura-Kamada, K., Matsuo, T., Yamasaki, T. and Minagawa, J. (2019) The CONSTANS flowering complex controls the protective response of photosynthesis in the green alga Chlamydomonas. Nat. Commun., 10, 4099.

Trissl, H.W. and Wilhelm, C. (1993) Why do thylakoid membranes from higher plants form grana stacks? Trends Biochem. Sci., 18, 415-419.

Troiano, J.M., Perozeni, F., Moya, R., Zuliani, L., Baek, K., Jin, E., Cazzaniga, S., Ballottari, M. and Schlau-Cohen, G.S. (2021) Identification of distinct $\mathrm{pH}$-and zeaxanthin-dependent quenching in LHCSR3 from Chlamydomonas reinhardtii. eLife, 10, e60383.

Wietrzynski, W., Schaffer, M., Tegunov, D., Albert, S., Kanazawa, A., Plitzko, J.M., Baumeister, W. and Engel, B.D. (2020) Charting the native architecture of Chlamydomonas thylakoid membranes with single-molecule precision. eLife, 9, e53740.

Wobbe, L., Bassi, R. and Kruse, O. (2016) Multi-level light capture control in plants and green algae. Trends Plant Sci., 21, 55-68.

Wood, W.H.J., Barnett, S.F.H., Flannery, S., Hunter, C.N. and Johnson, M.P. (2019) Dynamic thylakoid stacking is regulated by LHCII phosphorylation but not its interaction with PSI. Plant Physiol., 180, 2152-2166. 

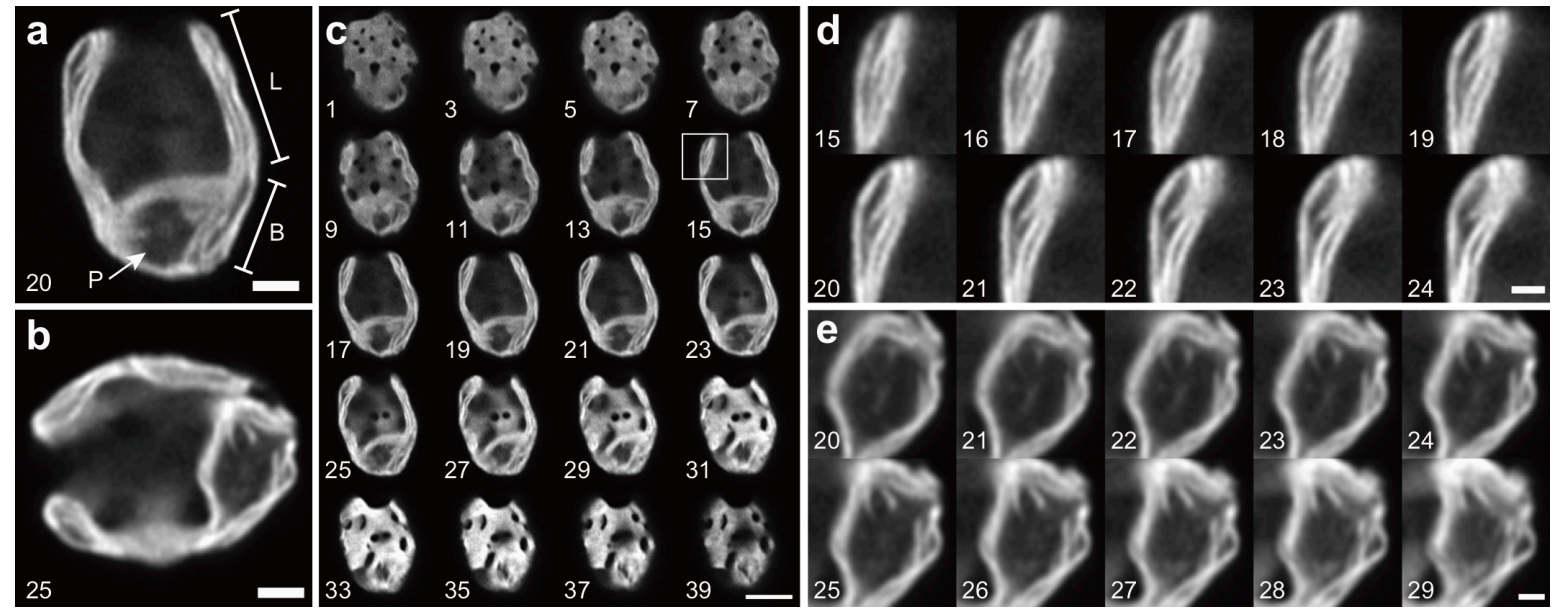

Figure 1. Airyscan images of $\boldsymbol{C}$. reinhardtii acclimated to LL. a, b. Representative images of the LLacclimated cells observed by $\mathrm{Chl}$ fluorescence. L, lobe; B, base; and P, pyrenoid. c. Optical serial sectioning of the cell (in a) through z-stack. d. Enlarged images for optical sectioning of a lobe region (a square in c), showing layers of thylakoid membranes. e. Enlarged images for optical sectioning of a pyrenoid region (in b), showing pyrenoid tubules (Engel et al., 2015). Numbers indicate slice numbers of z-stack images. Scale bars $=2 \mu \mathrm{m}(\mathbf{a}, \mathbf{b}), 5 \mu \mathrm{m}(\mathbf{c}), 1 \mu \mathrm{m}(\mathbf{d}, \mathbf{e})$. 

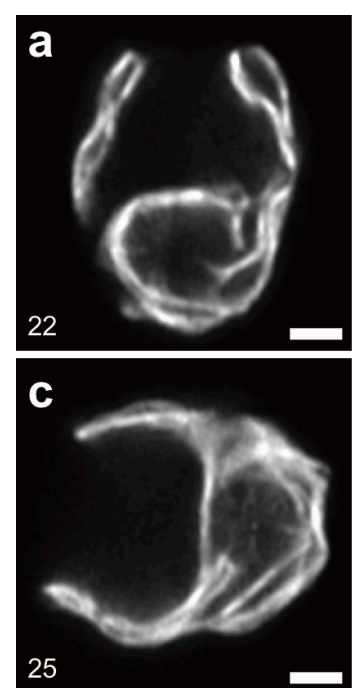

25
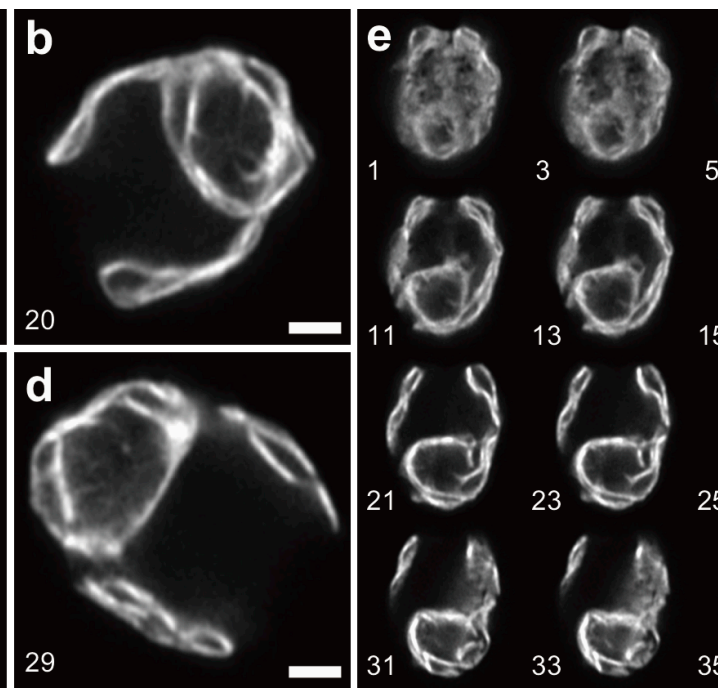

5
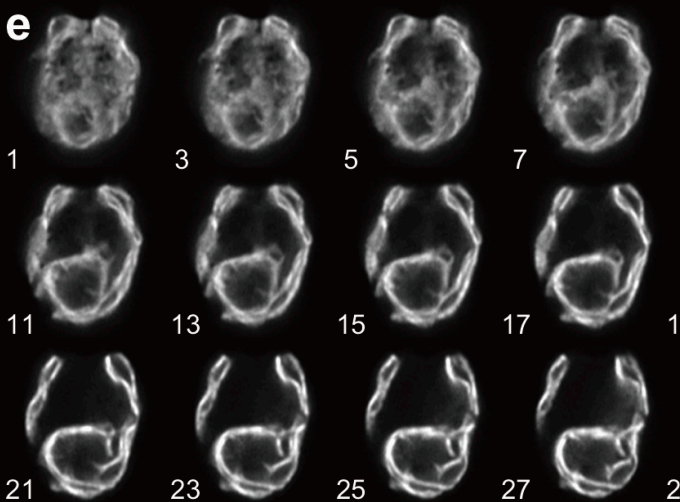

19

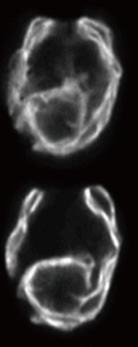

21

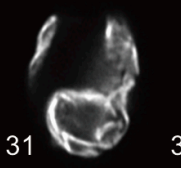

33
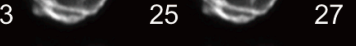

27

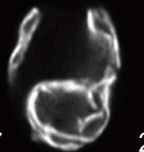

29
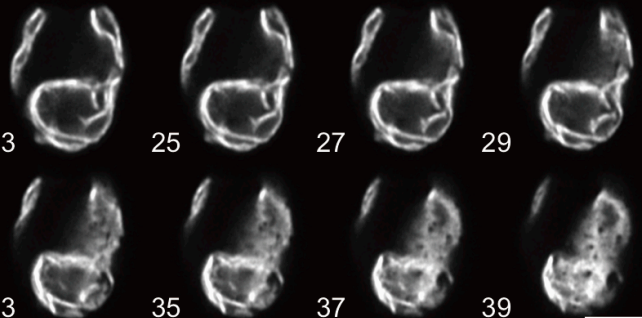

35
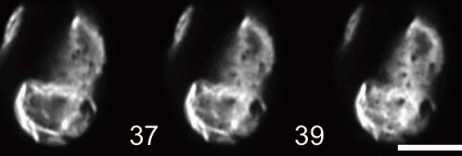

Figure 2. Airyscan images of $\boldsymbol{C}$. reinhardtii acclimated to HL. a-d. Representative images of the HLacclimated cells observed by $\mathrm{Chl}$ fluorescence. e. Optical serial sectioning of the cell (in a) through z-stack. Numbers indicate slice numbers of z-stack images. Scale bars $=2 \mu \mathrm{m}(\mathbf{a}-\mathbf{d}), 5 \mu \mathrm{m}(\mathbf{e})$. 
bioRxiv preprint doi: https://doi.org/10 1101/2021.06.26.450046; this version posted June 27, 2021. The copyright holder for this preprint (which was not certified by peer review) is the author/funder, who has granted bioRxiv a license to display the preprint in perpetuity. It is made available under aCC-BY-NC-ND 4.0 International license.
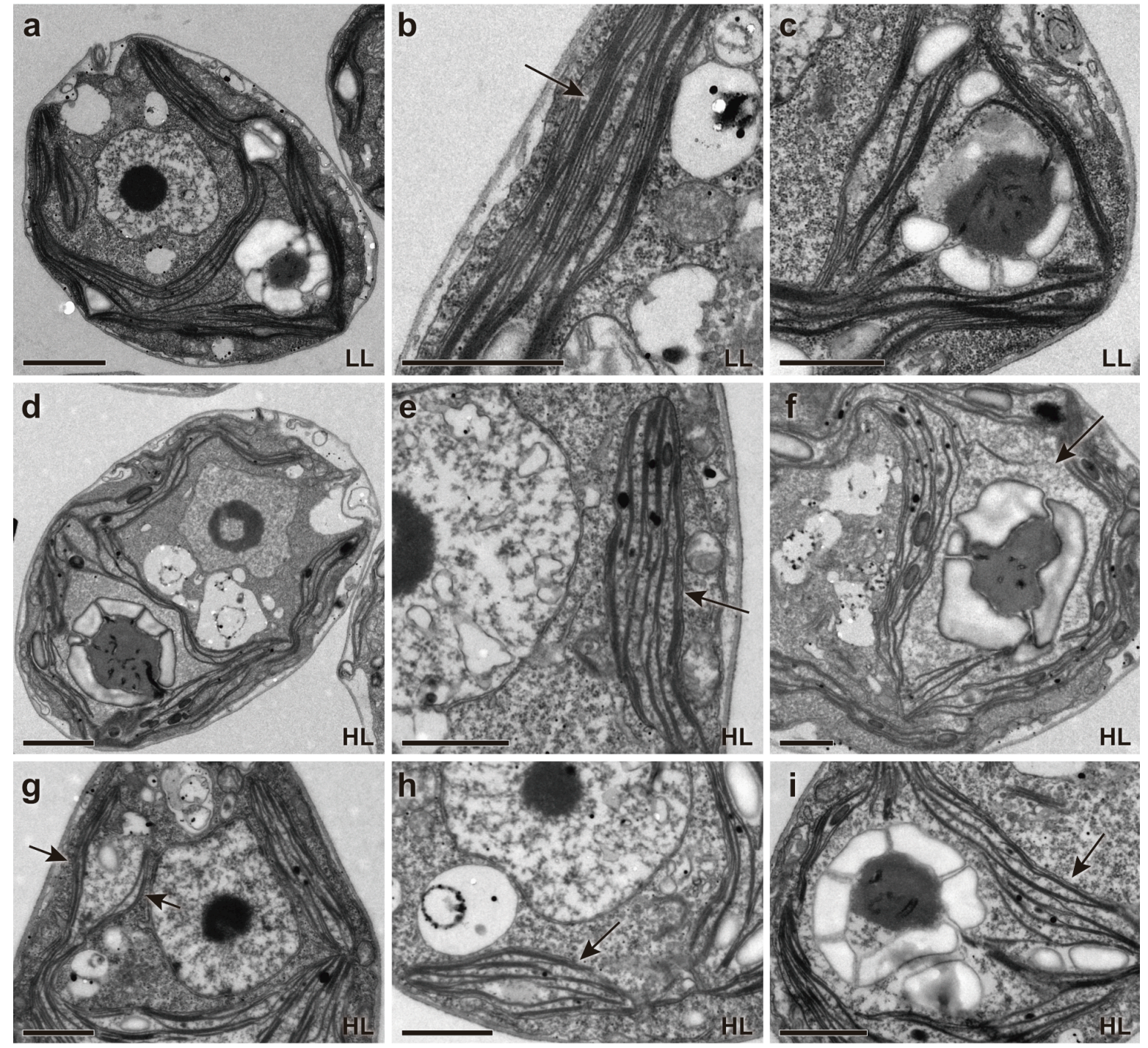

Figure 3. TEM images of $C$. reinhardtii acclimated to LL and HL. a-c. Representative images of the LLacclimated cells, showing an overall cell structure (a), thylakoid membranes (indicated by an arrow) at a lobe region (b), and a base region (c). Representative images of the HL-acclimated cells, showing an overall cell structure (d), thylakoid membranes (indicated by an arrow) at lobe regions (e, $\mathbf{g}, \mathbf{h}$ ), and enlarged stromal spaces (an arrow in f) around the pyrenoid at base regions (f, i). Scale bars $=2 \mu \mathrm{m}(\mathbf{a}, \mathbf{d}), 1 \mu \mathrm{m}(\mathbf{b}$, c, e-i). 

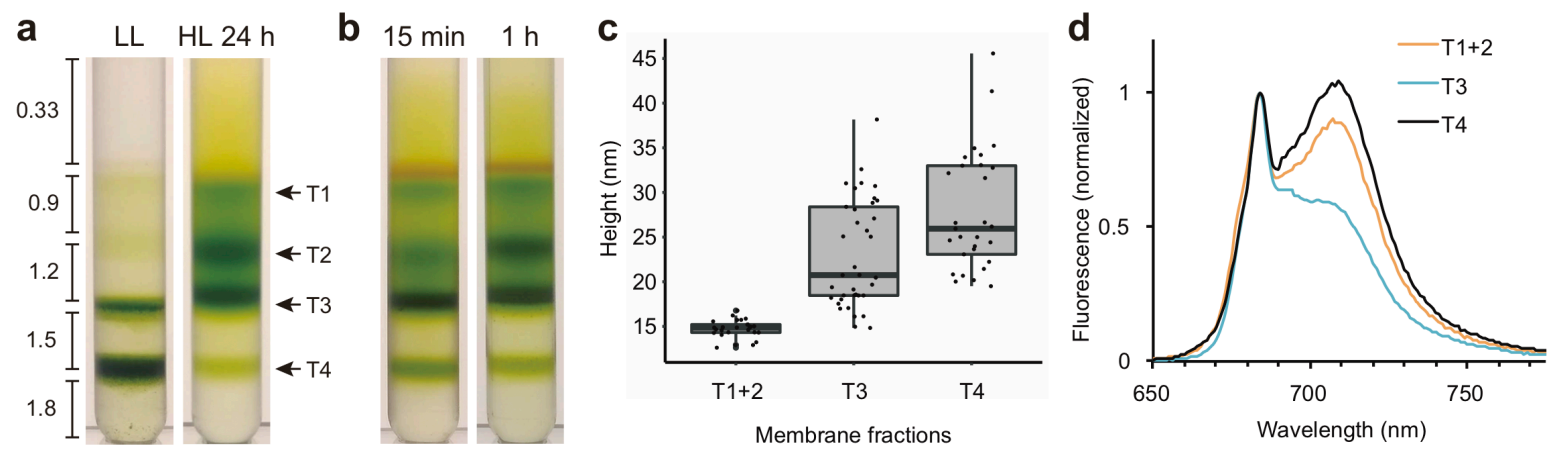

Figure 4. Analytical sucrose density-dependent membrane fractionation. a. The cells acclimated to LL and HL were gently disrupted (a total of $0.5 \mathrm{mg} \mathrm{Chl}$ ) and separated by sucrose step-gradient centrifugation. Numbers indicate the molar concentration of sucrose (see Methods for details). Different membrane fractions were indicated as T1-T4. b. The results for the cells treated under HL for $15 \mathrm{~min}$ and $1 \mathrm{~h}$. c. The height of isolated membranes in each fraction was measured by AFM. d. Chl fluorescence emission spectra at $77 \mathrm{~K}$. Spectra were normalized at $684 \mathrm{~nm}$. T1 and T2 $(\mathrm{T} 1+2)$ and T3 were obtained from HL samples, and T4 was obtained from LL samples. 

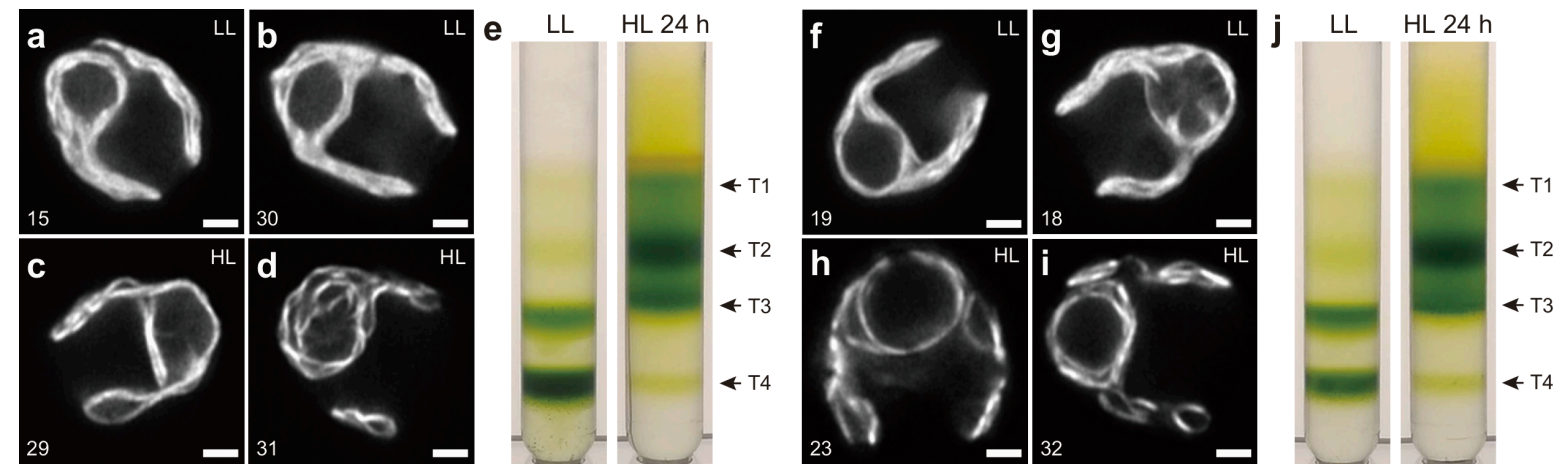

Figure 5. Thylakoid structural changes after HL acclimation in npq4 lhcsrl and spa1-1. Representative Airyscan images of the npq4 lhcsrl mutant cells acclimated to LL (a, b) and HL (c, d). e. Analytical sucrose density-dependent membrane fractionation using the npq4 lhcsrl mutant cells acclimated to LL and HL were gently disrupted (a total of $0.5 \mathrm{mg} \mathrm{Chl}$ ) and separated by sucrose step-gradient centrifugation (see Methods for details). Representative Airyscan images of the spa 1-1 mutant cells acclimated to LL (f, g) and HL (h, i). j. Analytical sucrose density-dependent membrane fractionation using the spal-1 mutant cells acclimated to LL and HL were gently disrupted (a total of $0.5 \mathrm{mg} \mathrm{Chl}$ ) and separated by sucrose stepgradient centrifugation (see Methods for details). Numbers (in a-d, f-i) indicate slice numbers of z-stack images. Scale bars $=2 \mu \mathrm{m}$. Different membrane fractions were indicated as T1-T4. 

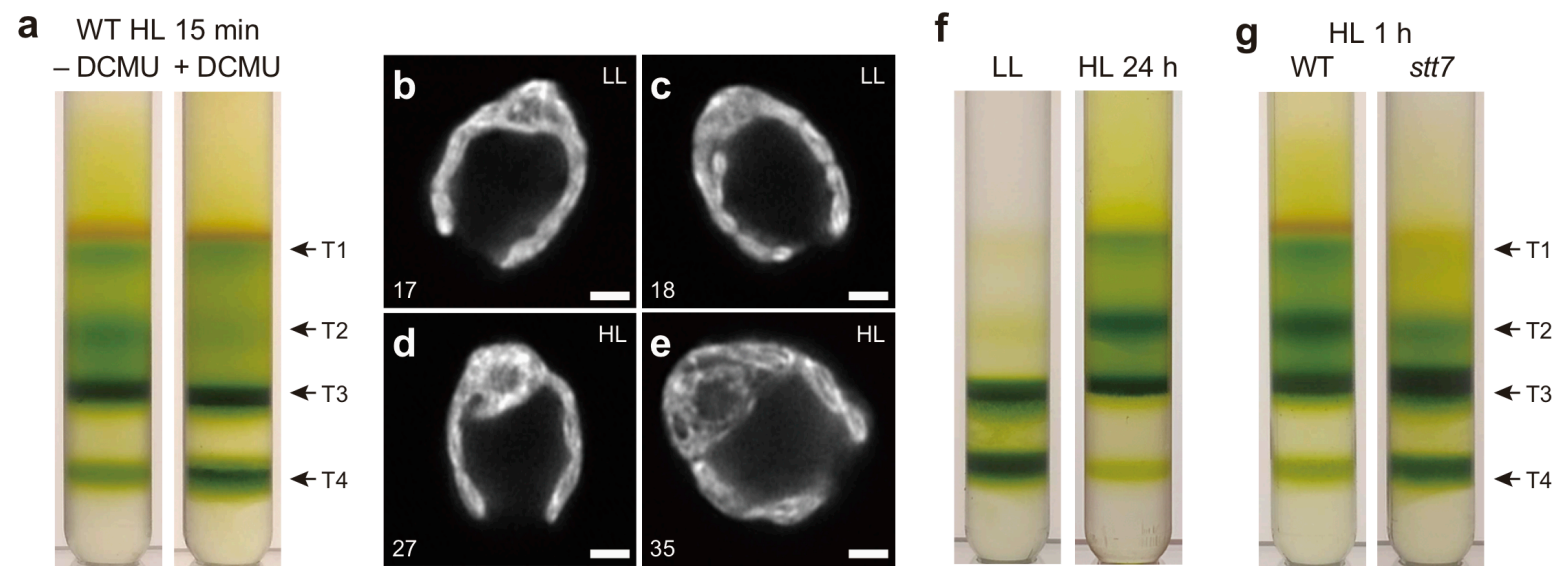

Figure 6. The effect of photosynthetic electron transfer and state transitions. a. Analytical sucrose density-dependent membrane fractionation using WT cells treated with DCMU during 15 min of HL treatment were gently disrupted (a total of $0.5 \mathrm{mg} \mathrm{Chl}$ ) and separated by sucrose step-gradient centrifugation (see Methods for details). Different membrane fractions were indicated as T1-T4. b. Representative Airyscan images of the stt7-9 mutant cells acclimated to LL (b, c) and HL (d, e). Numbers indicate slice numbers of z-stack images. Scale bars $=2 \mu \mathrm{m}$. f. Analytical sucrose density-dependent membrane fractionation using the stt7-9 mutant cells acclimated to LL and HL (for $24 \mathrm{~h}$ ) were gently disrupted (a total of $0.5 \mathrm{mg} \mathrm{Chl}$ ) and separated by sucrose step-gradient centrifugation. g. Analytical sucrose densitydependent membrane fractionation using the WT and stt7-9 mutant cells acclimated to HL for $1 \mathrm{~h}$ were gently disrupted (a total of $0.5 \mathrm{mg} \mathrm{Chl}$ ) and separated by sucrose step-gradient centrifugation. 
bioRxiv preprint doi: https://doi.org/10.1101/2021.06.26.450046; this version posted June 27, 2021. The copyright holder for this preprint (which was not certified by peer review) is the author/funder, who has granted bioRxiv a license to display the preprint in perpetuity. It is made available under aCC-BY-NC-ND 4.0 International license.

\section{SUPPORTING INFORMATION}

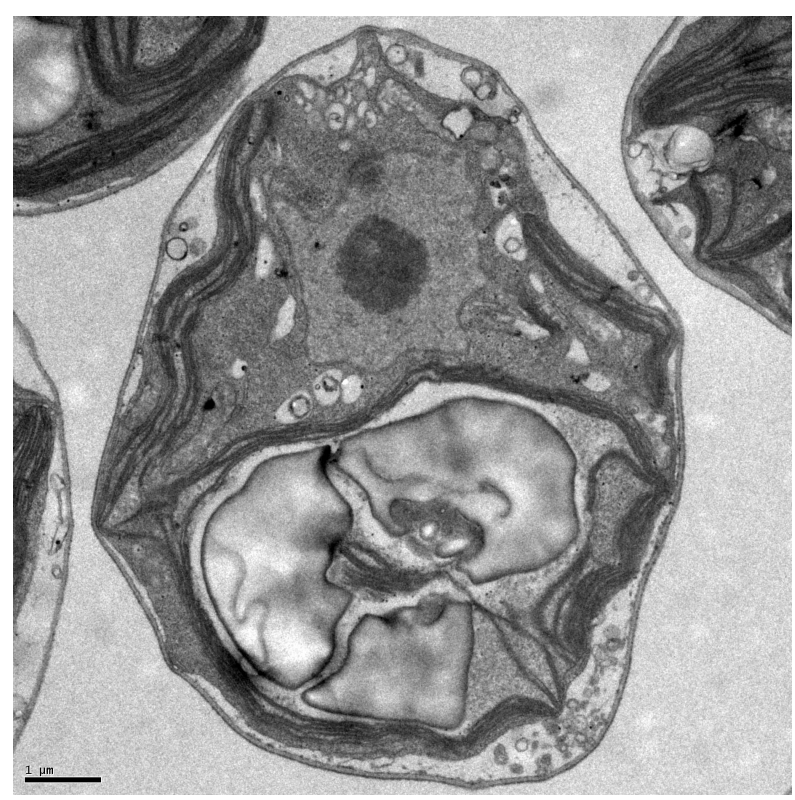

Figure S1. The representative TEM image of the $C$. reinhardtii spa1-1 cell acclimated to LL conditions. Scale bar $=1 \mu \mathrm{m}$. 